\title{
Aplicaciones basadas en los SIG para el diseño de itinerarios culturales en paisajes con valores patrimoniales difusos. El caso del territorio del Bajo Guadalquivir (Conjunto Arqueológico de Itálica): Revisión sistemática de literatura científica
}

\author{
Rebeca Merino del Río ${ }^{1}$, Antonio Tejedor Cabrera ${ }^{2}$, \\ Mercedes Linares Gómez del Pulgar ${ }^{3}$
}

Instituto Universitario de Arquitectura y Ciencias de la Construcción, Universidad de Sevilla E-mail: 'rmdelrio@us.es, ${ }^{2}$ atejedor@us.es, ${ }^{3}$ mercedeslgdp@us.es

\begin{abstract}
Resumen. En los últimos años, los sistemas de información geográfica (SIG) se han erigido como la herramienta clave para realizar análisis espaciales avanzados de diversa indole a escala territorial. Lo evidencia el elevado número de estudios y publicaciones de disciplinas dispares como la geografia, la arqueología, la ingeniería civil o el urbanismo. Sin embargo, su aplicación arquitectónica en la restauración y revalorización de paisajes con valores patrimoniales no ha sido suficientemente explorada desde un punto de vista cientifico. El Conjunto Arqueológico de Itálica, situado territorialmente en el Bajo Guadalquivir en el área metropolitana de Sevilla, forma parte de un amplio sistema de restos arqueológicos difusos actualmente desarticulado debido a la expansión frenética de los núcleos urbanos a costa del medio rural próximo. El itinerario cultural se presenta como una infraestructura de movilidad contemporánea capaz de ofrecer una lectura integral de los antiguos cursos naturales y sistemas de movilidad históricos. Su diseño asistido por los SIG, atendiendo a otros recursos medioambientales y servicios culturales, permite revalorizar zonas urbanas y periurbanas deprimidas, así como lograr un uso inclusivo del territorio. Esta comunicación examina una posible metodología de diseño de itinerarios culturales en paisajes patrimoniales con SIG a escala territorial basada en la revisión sistemática de la literatura cientifica más relevante. Concretamente, su aplicación se limita al entorno del Conjunto Arqueológico de Itálica. Esto nos permite evaluar la oportunidad de un estudio como el que se propone y detectar aquellas funcionalidades de los SIG más adecuadas para cada fase de la metodología.
\end{abstract}

Palabras clave: Aplicación, SIG, itinerario cultural, paisaje patrimonial, Itálica, revisión sistemática de literatura

El conjunto arqueológico de Itálica: introducción y reflexión sobre la pertinencia de una intervención a escala territorial

La monumental ciudad romana de Itálica, ubicada en el actual municipio de Santiponce, al noroeste de la ciudad de Sevilla, alcanzó su máxima plenitud en época del emperador Adriano. La vetus urbs, la antigua ciudad republicana que hoy en día se encuentra sepultada bajo la urbanización contemporánea, aflora en ciertos puntos del tejido urbano, evidenciando el noble origen de este municipio. La parte de la ciudad de época imperial, la nova urbs, se sitúa al norte de la vetus urbs. Esta es más fácilmente recreable gracias a las excavaciones y la protección conferida al conjunto arqueológico, y complementa a la 
ciudad republicana. El apogeo y decadencia de Itálica se puede vislumbrar al considerar su estratégica situación en el territorio del Bajo Guadalquivir y las interacciones económicas y simbólicas en el seno de la provincia más romanizada del Imperio fuera de la península itálica, a la que debe su nombre. Su evolución hasta época reciente dependió en gran medida de las infraestructuras de movilidad y de los cursos naturales de transporte en su área de influencia. Hacia el siglo II d.C., en el momento de mayor plenitud, la ciudad se situaba en un nodo de la red de comunicaciones del sur de la Bética que permitía hacer llegar el material procedente de las explotaciones mineras del valle del Guadalquivir hasta el océano por el rio Betis. Su abandono se estima que pudo verse forzado por la alteración del trazado del cauce y el calado del río. Como indica Borja Barrera y otros (2018) en época romana el cauce de dicho río transcurriría más próximo a Itálica y al lado este de la cornisa del Aljarafe. Con el paso de los siglos, las crecidas y depósito de sedimentos han ido alejando el cauce hacia el este, lo que finalmente hizo impracticable el traslado de materiales e impulsó el desarrollo de otros núcleos urbanos cercanos, como Hispalis o Caura.

Más allá de las sucesivas modificaciones de este territorio a lo largo de su historia, es durante la segunda mitad del siglo XX cuando se constata un mayor impacto del proceso de urbanización sobre el mundo rural. La ciudad de Sevilla y los núcleos urbanos adyacentes que hoy en día constituyen su área metropolitana - Santiponce entre ellos-, experimentaron un fuerte crecimiento a costa del suelo rural. En la figura 1, se representan Sevilla y los núcleos urbanos que constituyen su área metropolitana. Las distintas tonalidades reflejan los distintos paisajes: en un tono más claro, la vega del Guadalquivir; en tono intermedio, los campos de Gerena; y en una tonalidad más oscura, la comarca del Aljarafe. Precisamente, al tiempo que se produjo esta urbanización, numerosos restos arqueológicos fueron descubiertos, evidenciando la extraordinaria riqueza de la historia del territorio en el pasado. En el plano se representan con puntos y polígonos parte de los restos arqueológicos catalogados por el Instituto Andaluz del Patrimonio Histórico (esta información debe ser revisada y completada en un futuro). Estos restos difusos, pertenecientes en su mayoría a la Edad del Bronce y a la época romana alto-imperial, han permitido conocer la evolución de la zona desde época tartésica hasta nuestros días. El descontrolado crecimiento del área metropolitana hasta mediados de los años ochenta, difícilmente reversible, ha neutralizado cualquier opción de realizar una lectura integral de la historia del territorio, además de dificultar enormemente cualquier estrategia de restauración y fruición de los paisajes culturales.

\section{Algunas precisiones sobre los modos y las formas}

La definición formal y material de un proyecto, no deja de ser un proceso que subsume de manera sintética el método de trabajo de Taller al que acabamos de referirnos y que debe responder a dos aspectos que actualmente son ineludibles: que se pueda gestionar social $\mathrm{y}$ económicamente y que esté justificado ambientalmente.

El primer aspecto se basará en la estructura socioeconómica de referencia, considerada como una red, en cuyos nodos se situarán los distintos actores, receptores de los logros del proyecto e igualmente sufridores de sus deficiencias y desequilibrios. Considerar de forma adecuada el entramado de las administraciones, empresas, asociaciones ciudadanas y la propia ciudadanía como referente, será clave en la factibilidad del proyecto.

Los aspectos ambientales son hoy en día fundamentales en la valoración de los proyectos. Para la definición de la forma, será necesaria una adecuada configuración de la misma en relación con las preexistencias. Para el proceso de construcción, la adecuada gestión de los residuos y para las cuestiones energéticas, la minimización de las emisiones y del gasto energético de explotación de los espacios establecidos en el proyecto.

En los aspectos ambientales ligados a la forma, tendrá que establecerse aquellos modos o paradigmas culturales de referencia que sean más adecuados a los problemas planteados. En ese sentido nos referiremos a sistemas o 


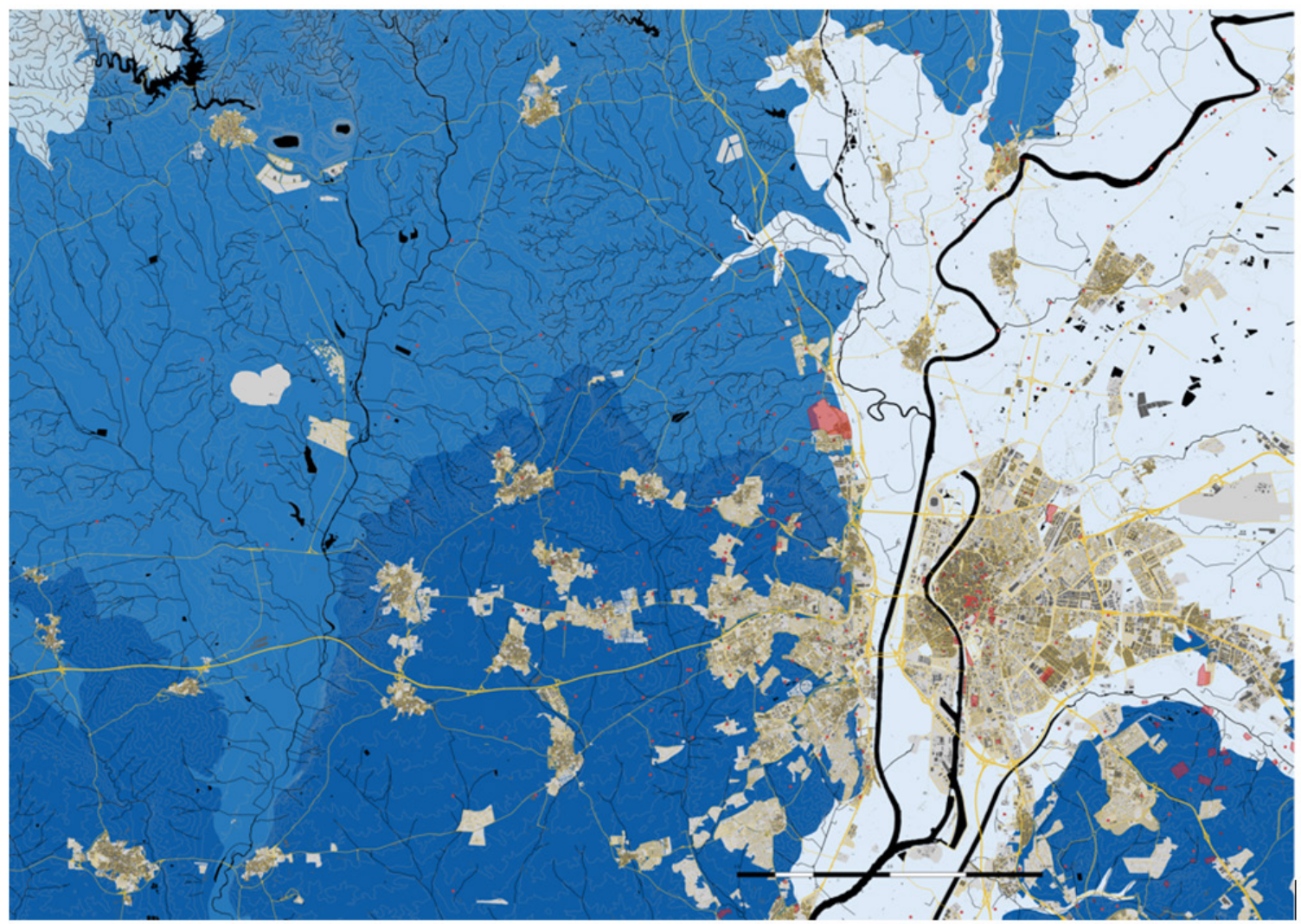

Fig. 1. Plano basado en los Sistemas de Información Geográfica donde se superponen los municipios del área metropolitana de Sevilla sobre el mapa de paisajes de Andalucía del DERA. Fuente: Autor, 2019.

códigos de asimilación a las formas urbanas preexistentes, asumiendo para la arquitectura actual su condición "ecléctica" y adoptando algunos referentes como el de la arquitectura urbana de Álvaro Siza Vieira que se presta muy ajustadamente a la manera en que el maestro contemporáneo utiliza sus conocimientos profundos de construcción y arquitectura, para adaptarse en cada situación a la correcta escala del entorno urbano en el que se sitúa su proyecto, como en un continuo ejercicio de restauración urbana y arquitectónica. (Kenneth Frampton, 1999)

\section{El itinerario cultural: instrumento de planeamiento ecológico del territorio}

La mejora y restauración del paisaje cultural no sólo hace accesible a la sociedad la historia del territorio. También tiene un impacto directo sobre la economía de la región y sobre su planificación urbanay territorial. En elConvenio Europeo del Paisaje de 2000 se anuncia una nueva tendencia que implica la utilización sostenible de los distintos recursos del territorio como base para la actividad turística Entre ellos destacamos el patrimonio arqueológico, arquitectónico y natural. Son numerosos los estudios que avalan el impacto económico positivo del turismo en la revitalización del medio rural (Esparcia, 2014; Gullino, Devecchi y Larcher, 2018; Horlings y Marsden, 2014). Así, la revalorización de los paisajes culturales contribuye a la oferta de servicios culturales de la región, diversificando la oferta turística y promoviendo el descongestionamiento de otras áreas con alta afluencia de visitantes. Por su lado, la restauración de paisajes culturales se convierte en una importante fuente de conocimiento para una planificación ecológica del entorno construido, más sensitiva a las relaciones entre los seres vivos entre sí y entre estos y el entorno físico.

En el caso particular de Itálica y su área de influencia, se estima que la restauración y mejora de los paisajes culturales contextualiza el yacimiento en el territorio - haciéndolo así más accesible (Alonso López, 2010)—, a la vez 
que contribuye a mejorar zonas actualmente deprimidas incorporándolas a un circuito con una posible explotación turística. El proyecto Smart Architectural and Archaeological Heritage: instrumentos y estrategias de innovación para la integración de la gestión patrimonial, turística y paisajística tiene como objetivo construir una ciencia del patrimonio que, mediante la aplicación de metodologías que superen los límites de las disciplinas tradicionales, se apoyen en el análisis espacial avanzado con herramientas basadas en los Sistemas de Información Geográfica (SIG). Con este objetivo se presenta el itinerario cultural como una estrategia concreta de intervención en el territorio, capaz de articular el patrimonio arqueológico inmueble difuso, desvelando para el gran público la sucesión de asentamientos humanos que a lo largo de la historia se han sucedido. El itinerario cultural promueve la comprensión integral de aquellos territorios con elevada carga histórica - como es el del bajo Guadalquivir a su paso por el área metropolitana de Sevilla-, a la vez que se estima que puede revitalizar zonas urbanas actualmente deprimidas por su situación o la falta de inversiones. En la figura 2, se puede observar la situación del borde urbano de Santiponce hacia el área protegida de Itálica. El crecimiento descontrolado de la ciudad ha sepultado numerosos restos inmuebles de época romana republicana y limita cualquier opción de restaurar estos paisajes culturales.

En las últimas décadas el uso de herramientas y aplicaciones de análisis espacial avanzado basadas en los SIG se ha generalizado entre los arquitectos y paisajistas (Purdum, 1999; Ball, 2002; Thompson y otros, 2011). Dada la escala territorial de la intervención del itinerario cultural, se ha considerado que las aplicaciones basadas en estos sistemas pueden ser de gran utilidad para asistir al arquitecto durante el proceso de diseño y planificación. Entre otras funcionalidades, permiten la visualización espacial de conjuntos de datos de naturalezas diversas o la realización de análisis multicriterio sobre un modelo de trabajo georreferenciado.

\section{Revisión sistemática de literatura científica: método y material}

Ante esta situación, resulta fundamental definir una metodología de trabajo que, aunque centrada en el caso concreto de Itálica, debe ser exportable a otros emplazamientos. Este protocolo de intervención en el territorio debe atender de manera específica a las posibilidades de los SIG en relación con la comprensión y ordenación de los paisajes culturales. La presente comunicación persigue esbozar una posible metodología de trabajo para diseñar itinerarios culturales en paisajes con alto valor patrimonial asistida por los Sistemas de Información Geográfica. Para ello, se realiza una revisión sistemática de la literatura científica producida a tal efecto indexada en las bases de datos con mayor impacto, en busca de aplicaciones y estrategias exportables a nuestro espacio de trabajo.

La revisión sistemática se realiza de



Fig. 2. Fotografía desde el conjunto arqueológico de Itálica hacia el municipio de Santiponce (a). Fotografía desde la A-8078 hacia el conjunto arqueológico de Itálica (b). Fuente: Autor, 2019. 
acuerdo con la metodología propuesta por Gough, Oliver y Thomas (2012; 2013), como ya hiciera con éxito Ferreira Lopes (2018). Los diez pasos que comprenden la metodología se estructuran en dos fases: una primera revisión cuantitativa y una segunda cualitativa. Por su limitada extensión, en esta comunicación relacionaremos los resultados arrojados tras aplicar los distintos criterios de exclusión y nos referiremos a la primera revisión de índole cuantitativa. Para extraer la muestra objeto de análisis cuantitativo, es necesario determinar una serie de parámetros y elementos de obtención. Los parámetros de búsqueda definitivos se fijan tras contrastar los resultados provisionales y reajustar los criterios y elementos iniciales. Una muestra válida es aquella formada por un número considerable de entradas que poseen una cierta heterogeneidad y es indicativa del estado de la cuestión constatado. Esta muestra es sometida a un análisis cuantitativo sobre la base que proporcionan los títulos, resúmenes y palabras claves.

Las bases de datos empleadas para realizar la revisión sistemática de la bibliografía son Web of Science (WOS) y Scopus, por su mayor impacto y alcance internacional. Se es consciente de que existe notable material publicado a tal respecto en libros y revistas indexadas en otras bases de datos. Sin embargo, y dado que también se pretende contemplar el estado de la cuestión y ponderar la oportunidad del tema propuesto, se entiende que la muestra obtenida en estos catálogos es suficientemente significativa para subrayar su idoneidad a nivel internacional y destilar aquellas aplicaciones de los SIG más extendidas en relación con el movimiento en el territorio y la restitución de paisajes culturales.

La búsqueda definitiva se basa en la combinación en grupos de tres en tres de cuatro elementos. Estos cuatro elementos seleccionados son: el término SIG; el componente archaeo*; la operación (route OR path) y la conjunción "cultural landscape". Se puede observar que, para ampliar la búsqueda a los términos 'archaeology' y 'archaeological' en los campos de búsqueda, se introduce el componente 'archaeo' seguido del signo '*' que dilata la indagación a todos los términos que lo incluyen. Inicialmente la búsqueda se restringió en el tercer elemento al término 'route', lo que en adelante se consideró insuficiente. Así, se realizó una segunda búsqueda sustituyendo este término por 'path', el elevado número de entradas diferentes evidenció la idoneidad de esta decisión. Se procedió a eliminar los elementos repetidos, tal y como habría operado en el buscador de las bases de datos la operación (route OR path).

\section{Revisión sistemática de literatura científica: descripción de resultados}

La búsqueda en Web of Science arroja un total de 165 resultados, de los cuales 32 entradas se encuentran repetidas, 12 son inaccesibles desde la Universidad de Sevilla y 26 no son considerados porque la temática se aleja en exceso de los intereses de la investigación. Para ello se ha contrastado el contenido de los resúmenes disponibles en las bases de datos, lo que ha resultado en un total de 95 artículos y comunicaciones procedentes de Web of Science considerados para un análisis posterior. La búsqueda en Scopus arroja un total de 195 resultados, de los cuales, tras contrastarlos entre sí y con los resultados procedentes de Web of Science, se han eliminado 115 por estar duplicados, 13 por ser inaccesibles y 26 por la dispersión temática. Se aíslan para un análisis posterior un total de 41 artículos y comunicaciones procedentes de Scopus.

Las cifras globales son 136 entradas consideradas para su revisión - lo que parece una muestra razonable para un estudio detallado de contenido-, 52 entradas no consideradas porque la temática se aleja del sujeto de estudio, 25 entradas no consideradas porque el contenido es inteligible o por ser inaccesibles con los medios disponibles en la Universidad de Sevilla y 147 artículos eliminados por estar repetidos.

Con la revisión sistemática de los resúmenes se procede a una catalogación de los artículos y comunicaciones de acuerdo con las ramas de conocimiento donde se engloba la investigación y el tipo de estudio que se plantea. Ello nos permite, por un lado, detectar aquellas ramas en las que hay un mayor número de investigaciones relacionadas con el 
tema propuesto y, por otro, valorar qué tipos de estudios son mayoritarios en relación con la movilidad y el análisis de redes y flujos en el territorio.

En la primera categoría sobre la rama de conocimiento a la que pertenecen los artículos, la evaluación de los títulos, resúmenes y palabras claves arroja las siguientes cifras: 17 entradas pertenecen a la disciplina de la arquitectura-urbanismo, 5 a las ciencias naturales, 4 a las ciencias sociales, 98 al área de las humanidades y 12 a la rama de ingeniería. La segunda categoría objeto de revisión cuantitativa constata el tipo de estudio que se organiza en dos subcategorías: los estudios de corte histórico, con 94 entradas, y los estudios orientados al diseño y gestión de paisajes culturales, con 42 entradas.

\section{Revisión sistemática de literatura científica: discusión y análisis cuantitativo}

Destaca la proporción mayoritaria de artículos científicos procedentes del área de las humanidades, concretamente, de la arqueología. La significativa, pero relativamente escasa, colección de artículos que podrían englobarse en las disciplinas de la arquitectura, el urbanismo y/o la ingeniería civil, puede deberse, entre otras razones, a que el número de revistas temáticas indexadas es menor y aún se está consolidando la incorporación de estas materias al mundo de las publicaciones científicas. Entre los artículos descartados por poseer una temática demasiado dispar, se encuentran un gran número de estudios de ciencias naturales que tratan de analizar la evolución de "paisajes culturales" primitivos en los que la presencia humana no es determinante ni su finalidad última.

La existencia de un mayor número de estudios de corte historiográfico nos permite aislar una de las principales finalidades de los SIG en investigaciones sobre paisajes culturales, la corroboración de hipótesis sobre los análisis espaciales avanzados. Así, se observan dos tendencias diferenciadas que atienden a la finalidad del estudio. La primera vertiente, mayoritaria, es la que trata de profundizar y complementar el conocimiento histórico. El análisis espacial avanzado por medio de extensiones de cálculo de caminos de mínimo coste, simplemente tomando como base el modelo del terreno elevado actual (DEM) - una base de trabajo que se entiende válida por su escasa variabilidad con el paso del tiempo (Herzog, 2014)—, permite aceptar o refutar hipótesis existentes o incluso plantear otras nuevas. Otros factores que se incorporan en estos estudios son las barreras que suponen las cuencas inundables, la cobertura vegetal o los usos del suelo, sin embargo, los dos últimos están sujetos a una mayor variabilidad y es difícil conocer cómo afectaban al movimiento en el pasado. Estas aplicaciones son típicas de los estudios de historiadores, arqueólogos, geólogos y/o antropólogos, es decir, del área de las humanidades. Se distingue un segundo uso del software basado en los SIG más orientado al diseño y gestión a escala urbana y/o territorial. Este tipo de estudios pertenece, en gran parte, a las áreas técnicas como la arquitectura, el urbanismo o la ingeniería civil. En estos casos, el análisis espacial avanzado se emplea para realizar cálculos complejos que asisten al arquitecto-urbanista en la toma de decisiones. Entre las extensiones más empleadas destacamos el cálculo de rutas de mínimo coste, pero también el módulo que permite reclasificar conjuntos de datos y asignarles nuevos valores (indicadores) o la calculadora ráster para realizar cálculos espaciales con conjuntos de datos georreferenciados.

\section{Consideraciones finales sobre la pertinencia de la investigación y la metodología propuesta}

Aunque la finalidad de este estudio es esbozar una posible metodología de trabajo sobre la base de la revisión sistemática de la literatura científica, los resultados también nos permiten conocer el estado del arte. Las conclusiones se estructuran así en dos apartados: la oportunidad de un tema como el que se propone y la descripción de un posible protocolo de actuación para diseñar itinerarios culturales asistido por software basado en los SIG.

Se concluye que, habida cuenta de la escasez de trabajos que estudian de manera general la 
movilidad como estrategia en favor de una mayor accesibilidad al patrimonio arqueológico inmueble y base de una planificación ecológica del territorio, una investigación como la que se propone resulta pertinente. Que sepamos, ningún estudio se ha realizado hasta la fecha cuya finalidad sea la aproximación metodológica al diseño de itinerarios culturales para revalorizar paisajes con alto valor patrimonial. La revisión sistemática de la literatura en Web of Science y Scopus arroja únicamente dos estudios apoyados en los SIG sobre los paisajes culturales en el bajo Guadalquivir. Esto contrasta con las numerosas monografías y estudios específicos sobre el patrimonio mueble e inmueble en esta región publicados en libros y revistas no indexados, que deben ser catalogados en una primera fase del diseño de itinerarios en el caso concreto del área de influencia del conjunto arqueológico de Itálica.

El diseño de la metodología contempla, en primer lugar, la doble finalidad restauradora y revitalizadora del itinerario cultural. También considera la doble aplicación de los SIG en relación con los paisajes culturales - como complemento al estudio histórico y como software de apoyo en el diseño de infraestructuras - que se extrae al clasificar los resultados de acuerdo con el tipo de estudio. Se observa que en los artículos de corte histórico donde se aplican los SIG, antes del empleo del software se incluye un capítulo donde se presenta el estado de la cuestión y la hipótesis objeto de revisión. Así las cosas, se propone una metodología de trabajo en tres fases que tiene en cuenta estas cuestiones. Se parte de un proceso de documentación bibliográfica, de análisis de mapas y planos y de sistematización de la información histórica. Esta primera fase, por el carácter dinámico del itinerario cultural, se centra de manera concreta en las infraestructuras de movilidad y los cursos naturales, si bien la definición de las categorías específicas debe ser objeto de una reflexión más profunda. En una segunda fase, las hipótesis defendidas sobre la movilidad en el territorio se contrastan con el análisis espacial avanzado que resulta del uso del software basado en los SIG. Ello permite una mayor comprensión de los paisajes culturales a restaurar, así como reconocer y situar algunos de los restos inmuebles difusos catalogados como parte de una red histórica de movimiento. Se incorpora así la primera utilidad de los SIG, en una fase con una marcada finalidad de revisión crítica histórica. Para concluir, la tercera fase de trabajo tiene un carácter propositivo. Los SIG se emplean en su vertiente de asistencia al diseño del itinerario cultural, incorporando los significados históricos extraídos en la segunda fase y los distintos servicios destinados a satisfacer las demandas actuales de la sociedad.

\section{Agradecimientos:}

Esta investigación se enmarca en el Proyecto de Investigación financiado por el Ministerio de Ciencia, Innovación y Universidades "Smart Architectural and Archaeological Heritage: instrumentos y estrategias de innovación para la integración de la gestión patrimonial, turística y paisajística" coordinado por el Prof. Dr. Antonio Tejedor Cabrera (Instituto Universitario de Arquitectura y Ciencias de la Construcción, Universidad de Sevilla).

\section{Referencias}

Alonso López, Fernando. 2010. "Justificación de la necesidad de accesibilidad universal en los bienes de interés cultural". ACE: Arquitectura, Ciudad y Entorno 5(13): 1340. DOI: 10.5821/ace.v5i13.2491.

Ball, Jonathan. 2002. "Towards a Methodology for Mapping 'Regions for Sustainability' Using PPGIS". Progress in Planning 58(0): 81-140. DOI: $10.1016 / \mathrm{S} 0305-$ 9006(02)00020-X.

Borja Barrera, Francisco, César Borja Barrera, Á. Jiménez Sancho, y E. García Vargas. 2018. "Evolución de la llanura aluvial del bajo Guadalquivir durante el Holoceno mediosuperior. Geoarqueología y reconstrucción paleogeográfica de la vega de Itálica (Sevilla, España)". Boletín Geológico y Minero 129(1-2): 371-420. DOI: 10.21701/ bolgeomin.129.1.015.

Esparcia, Javier. 2014. "Innovation and Networks in Rural Areas. An Analysis from 
European Innovative Projects". Journal of Rural Studies 34(0): 1-14. DOI: 10.1016/j. jrurstud.2013.12.004.

Ferreira Lopes, P. 2018. "Achieving the State of Research Pertaining to GIS Applications for Cultural Heritage by a Systematic Literature Review". The International Archives of the Photogrammetry, Remote Sensing and Spatial Information Sciences, XLII-4: 169175.

Gough, D., S. Oliver, y J. Thomas. 2012. An Introduction to Systematic Reviews. Londres: SAGE Publications.

Gough, D., S. Oliver, y J. Thomas. 2013. Learning from Research: Systematic Reviews for Informing Policy Decisions: A Quick Guide. Londres: Nesta.

Gullino, Paola, Marco Devecchi, y Federica Larcher. 2018. "How can Different Stakeholders Contribute to Rural Landscape Planning Policy? The Case Study of Pralormo Municipality (Italy)". Journal of Rural Studies 57(0): 99-109. DOI: 10.1016/j. jrurstud.2017.12.002.

Herzog, Irmela. 2014. "Least-Cost Paths Some Methodological Issues". Internet Archaeology 0(36). DOI: 10.11141/ia.36.5.

Horlings, Lummina G., y Terry K. Marsden. 2014. "Exploring the 'New Rural Paradigm' in Europe: Eco-Economic Strategies as a Counterforce to the Global Competitiveness Agenda". European Urban and Regional Studies 21(1): 4-20. DOI: 10.1177/0969776412441934.

Purdum, Gary E. 1999. "GIS in Site Design: New Tools for Design Professionals". Landscape Journal 18(1): 107-8.

Thompson, Aaron W., Linda Stalker Prokopy, Kristin Floress, y Denise C. Weinkauf. 2011. "A Method for Incorporating Community Priorities into GIS: Challenges, Choices, and Directions for Landscape Planners". Landscape Journal 30(2): 299-312. DOI: 10.3368/lj.30.2.299. 